\title{
Philonsorbonne
}

$7 \mid 2013$

Année 2012-2013

\section{"La fête du trésor caché ». Spinoza dans la poétique d'Elsa Morante}

\section{Cristina SANTINELLI}

\section{Q OpenEdition}

\section{$\checkmark$ Journals}

\section{Édition électronique}

URL : https://journals.openedition.org/philonsorbonne/512

DOI : $10.4000 /$ philonsorbonne. 512

ISSN : 2270-7336

Éditeur

Publications de la Sorbonne

\section{Édition imprimée}

Date de publication : 15 novembre 2013

Pagination : 147-169

ISSN : 1255-183X

\section{Référence électronique}

Cristina SANTINELLI, « «La fête du trésor caché ». Spinoza dans la poétique d'Elsa Morante »,

Philonsorbonne [En ligne], 7 | 2013, mis en ligne le 18 décembre 2013, consulté le 08 juin 2021. URL http://journals.openedition.org/philonsorbonne/512; DOI : https://doi.org/10.4000/philonsorbonne. 512

(c) Tous droits réservés 


\title{
«La fête du trésor caché » Spinoza dans la poétique d'Elsa Morante ${ }^{(*)}$
}

\author{
Cristina Santinelli
}

\section{Réalisme et révolution}

Dans un petit essai aux tons presque lyriques, Elsa Morante exprime symboliquement l'amour profond pour sa ville, Rome, par un éloge singulier, plein d'affection et d'ironie, de Piazza Navona : «peu de Terriens, je crois - écrit-elle - peuvent autant que moi se vanter d'avoir visité et fréquenté toutes les plus belles places du monde», mais Piazza Navona demeure «la place centrale de l'univers», orgueilleusement vitale et accueillante, «située dans l'absolu de la réalité, hors de la dimension du temps » expression d'un «Baroque » qui «ne s'épuise pas à l'intérieur d'une période historique, mais c'est un élément réel de la nature et de l'imagination, nécessaire à la plénitude de la vie » ${ }^{1}$.

Le terme «baroque », dont elle précise ici la portée universelle, peut définir son écriture : fastueuse et plastique en même temps, soucieuse du détail, mais extrêmement pure et fluide, didactique et à la fois, captivante, réaliste et onirique ${ }^{2}-$ qui est «nécessairement » enracinée dans l'histoire et

(*). Il s'agit de la réélaboration d'une leçon faite à la Sorbonne à Paris le 2 mai 2012 au sein du séminaire coordonné par Chantal Jaquet, Pascal Sévérac et Ariel Suhamy, consacré à Spinoza, personnage littéraire. Une version en italien, avec quelques variantes de contenu et de disposition, a été publiée sous le titre : "Poetica della "festa" e filosofia della "gioia". Elsa Morante e Spinoza », dans la revue La Cultura (année L, n 3, décembre 2012, p. 443- 466).

1. E. Morante, Ma Navone [Navona mia, 1962], en Id., Pour ou contre la bombe atomique, p. 81, 84-86, traduction de J.-N. Schifano [éd. orig. : Pro o contro la bomba atomica, Milano, Adelphi, 1987, p. 77, 79-81].

2. G. Ferroni, Alla ricerca dell' "ultimo" libro, in Aa.Vv., Per Elsa Morante, Milano, Linea d'Ombra, 1993, p. 46 ; A. Berardinelli, Il sogno della cattedrale. Elsa Morante e il romanzo come archetipo, ivi, p. 28 et 14). 
la géographie de son temps ${ }^{3}$, et qui toutefois se place dans une perspective d'éternité, au-delà du temps : «le vrai poète - écrit-elle - sent [...] que nombre de ses lecteurs doivent encore naître, et que sa réalité est vraie à jamais $»^{4}$.

Elsa Morante (Rome, 1912-1985) a ressuscité, ou du moins repris, un genre agonisant dans la littérature italienne du $\mathrm{XX}^{\mathrm{e}}$ siècle : le roman, compris en tant que construction épique, ample, de grande envergure, aux grandioses structures portantes, mais extrêmement soignée dans les détails aussi ${ }^{5}$.

La puissance narrative, l'amour du détail et surtout l'efficacité incomparable des descriptions des paysages de l'âme - qu'elle est capable de sonder dans ses plis les plus profonds -, ont contribué, à juste titre, au rapprochement d'Elsa Morante des grands narrateurs de la seconde moitié du XIX ${ }^{\mathrm{e}}$ siècle, tout particulièrement de Dostoïevski et de Tolstoï. Dans son essai Sur le roman, une de ses très rares réflexions «théoriques » sur l'art d'écrire, Elsa Morante note que "Chaque drame humain», avant d'être social, « est un drame psychologique », et donc le romancier doit raconter la manière dont l'homme se rapporte aux choses ${ }^{6}$. C'est pourquoi seulement à travers la minutieuse reconstruction des psychologies des personnages, elle mettra en scène la complexité des grands thèmes sociaux tels que le pouvoir, la question méridionale, la maternité, l'exclusion, et bien d'autres encore comme les questions politiques, idéologiques, etc.

Personnalité complexe, rebelle et provocatrice - on l'a définie une personne "gaie, libre, anarchiste comme un oiseau ", mais aussi "austère comme les Tables de la Loi $\gg^{7}-$, dès ses premières expériences littéraires, Elsa Morante se révèle réfractaire à tout type d'homologation, de mode, de goût et de «tradition consacrée ${ }^{8}$, et sa puissance narrative, exubérante et irréductible, échappe à toute classification.

L'indépendance de son écriture vis-à-vis des «modèles »" n'exclut pas toutefois de nombreuses sources d'inspiration. Elle-même nous parle de ses

3. «Bref, un vrai romancier [...] communiquera, en outre, toujours nécessairement, aux générations contemporaines et futures, les plus sûres vérités sur "le lieu géographique" et sur "le temps historique" où il a vécu sa propre expérience humaine ». (E. Morante, Sur le roman [Sul romanzo,1959], en Id., Pour ou contre la bombe atomique, tr. cit., p. 62-63 ; éd. it. cit., p. 61 ; c'est moi qui souligne).

4. Ivi, tr. cit., p. 62 (éd. it. cit., p. 60).

5. A. Berardinelli, art. cit., p. 27 et 32.

6. Avec Tolstoï, elle affirme qu' «il est permis d'inventer tout ce qu'on veut dans un roman, sauf la psychologie ». "Pour moi - commente Elsa Morante - c'est la seule loi absolue du réalisme dans le roman » (E. Morante, Sur le roman, tr. cit., p. 52 ; éd. it. cit., p. 51).

7. F. Ramondino, La più bella dichiarazione?, in Aa.Vv., Festa per Elsa, a cura di G. Fofi e A. Sofri, Palermo, Sellerio, 2011, p. 47.

8. C. Garboli, Il gioco segreto. Nove immagini di Elsa Morante, Milano, Adelphi, 1995, p. 19. 9. «Exotique et familière, naturelle et hyperbolique, l'écriture de la Morante ne laisse entrevoir aucun modèle [...]. Elle est née d'elle-même » (ivi, p. 67-68). Toutes les traductions sont de l'auteur de l'article à moins qu'un autre traducteur ne soit mentionné. 
lectures et, surtout, précise ses passions littéraires : «Homère ; Cervantès ; Stendhal; Melville; Tchekhov et Verga»; ce sont ses écrivains (ou «poètes ») préférés, parce que plus que tous les autres - écrit-elle - ils «provoquent toujours en moi, à les fréquenter, une augmentation extraordinaire de vitalité. Au point que, plus d'une fois dans le cours [...] de mon histoire, je crois avoir été directement ressuscitée des morts, grâce à leur vertu $»^{10}$.

À côté des «grands » écrivains, il faut ajouter les compositeurs de musique classique - Mozart tout d'abord ${ }^{11}$ - et les philosophes : de Platon à Simone Weil en passant par la pensée orientale. Refusant toute distinction de genre, elle parle simplement de «chefs-d'œuvre ${ }^{12}$, définis en tant qu'expression de l'identité qu'il y a entre la beauté et l'éthique, identité capable de causer une augmentation de vitalité dans celui qui jouit de l'œuvre d'art : la musique de Mozart est «éthique », les Dialogues de Platon sont « beaux », et vice-versa, tous les deux font « ressusciter des morts ».

Les «chefs-d'œuvre» sont donc les œuvres littéraires, musicales, philosophiques dans lesquelles l'auteur, soutenu par la seule recherche de la vérité, s'exprime en toute sincérité, sans rechercher aucun intérêt, ni obéir à aucun conditionnement extérieur. Pour le romancier, donc, raconter ne signifie ni professer une «idéologie », ni assumer la réalité dans un schéma « idéal », et donc préjudiciel, mais - écrit-elle - c'est « faire le silence autour de soi et se libérer de tout écran culturel, de tout fétiche, de tout vice conformiste ${ }^{13}$, devenir la voix du monde, la puissance expressive des choses mêmes, à travers la recherche impartiale de la «vérité » qui produit spontanément la «beauté ».

Les « chefs-d'œuvre » - révèle Elsa Morante - constituent la véritable « révolution» permanente de l'humanité, parce qu'ils expriment la libertà dello spirito, la liberté de l'esprit. Ce terme n'indique pas une «entité métaphysique-éthérée », mais la realtà integra, propria e naturale dell'uomo, la dimension intègre et naturelle propre à l'homme dans son entier $^{14}$. La clé de la narrative de l'écrivaine est à rechercher donc dans cette attribution d'une nature intimement « révolutionnaire » à l'expression artistique, comprise en tant que déclinaison de la réalité intégrale de l'homme.

10. E. Morante, Sur le roman, cit., p. 78 (éd. it. cit., p. 73).

11. Sur le rapport d'Elsa Morante avec la musique, voir C. Samonà, «Elsa Morante e la musica », Paragone, ${ }^{\circ}$ 432, février 1986, p. 13-20.

12. «[...] quel air pur, quelle compagnie vraiment aristocratique que celle des chefsd'œuvre », écrit Morante le 16 mars 1938 (E. Morante, Diario 1938, Torino, Einaudi, 1989, p. 51).

13. E. Morante, Sur le roman, cit., p. 57 (éd. it. cit., p. 55).

14. E. Morante, Petit Manifeste des communistes (sans classe ni parti), Paris, Éditions Payot \& Rivages, 2005, p. 10 [éd. orig. : Piccolo manifesto dei comunisti (senza classe né partito), Roma, I sassi nottetempo, 2004, p. 7-9]. 
Les romans d'Elsa Morante sont toujours nouveaux et originaux quant à la reconstitution historique, à l'époque et aux personnages, mais si on les considère dans leur ensemble, on voit qu'ils couvrent et racontent l'histoire de tout le $X X X^{\mathrm{e}}$ siècle $^{15}$. Cette histoire s'y dessine en général, comme l'histoire d'une évolution, anthropologique aussi, de la condition humaine vers des horizons inédits d'aliénation et de perte de soi. La romancière est capable de les examiner dans leur tragicité, grâce à une grande puissance d'imagination, transfiguratrice, je dirais même onirique. Toutefois sa «prodigieuse imagination» n'est jamais fin en soi, ne poursuit point l'amour du fabuleux, mais elle se révèle « un moyen pour demeurer soudée et fidèle à la réalité », pour « être présente et vigilante » ${ }^{16}$.

«Un vrai roman - écrivait la narratrice - est toujours réaliste : fût-ce le plus fabuleux ! $»^{17}$. Mais il faut dissiper une équivoque sur le terme « réalisme», qui ne signifie ni reproduction photographique ni documentarisme, mais plutôt : simpatia con la realtà, sympathie avec la réalité $^{18}$. La « réalité »- dit Elsa Morante - ne doit pas être confondue avec la simple apparence (être là) des choses, mais doit être comprise « dans toute sa plénitude et sa richesse », à savoir "comme la valeur toujours vive et intègre qui est cachée dans les choses ${ }^{19}$. La réalité - écrit-elle encore «est d'une richesse inépuisable», parce qu' «elle se renouvelle et se multiplie pour chaque nouvelle créature vivante qui part l'explorer », c'est au romancier de transformer cette exploration, cette expérience contingente, « en une vérité poétique incorruptible ${ }^{20}$.

Voilà pourquoi Elsa Morante est reconnue comme un des rares narrateurs authentiques de la littérature italienne du $\mathrm{XX}^{\mathrm{e}}$ siècle, capable de donner une épaisseur philosophique ${ }^{21}$ au roman. On a alors défini le roman «le temple dans lequel se déroulent les rites de la reconnaissance de soi et de la conquête de la conscience », "l'instrument d'une méditation transcendantale sur le présent $»^{22}$.

15. C. Garboli, op. cit., p. 24-25.

16. F. Ramondino, art. cit., p. 47, voir aussi G. Fofi, All'analfabeta per cui scrisse, in Aa.Vv., Festa per Elsa, cit., p. 14. Faire face à la réalité, ne pas l'esquiver, c'était une conviction d'Elsa Morante, mais aussi une caractéristique de sa personnalité : «Tout ce qu'on peut dire d'elle, synthétiquement, c'est ceci : [...] qu'elle est allée jusqu'au fin fond des choses et des pensées [...]. Elle était extrême» (R. La Capria, La felicità e l'orrore, in Aa.Vv., Festa per Elsa, cit., p. 93).

17. E. Morante, Sur le roman, tr. cit., p. 51 (éd. it. cit., p. 50-51).

18. E. Morante, Il poeta di tutta la vita [1957], in Id., Pro o contro la bomba atomica, cit., p. 38 (tr. fr., Le poète de toute la vie, en Id., Pour ou contre la bombe atomique, cit., p. 152).

19. E. Morante, Le monde sauvé par les gamins, trad. par J.-N. Schifano, Note introductive, p. 9 (éd. orig. : Il mondo salvato dai ragazzini, Einaudi, Torino, 1968, Nota introduttiva, p. V).

20. E. Morante, Sur le roman, cit., p. 50 (éd. it. cit., p. 49-50).

21. C. D'Angeli, Leggere Elsa Morante. Aracoeli, La storia e Il mondo salvato dai ragazzini, Roma, Carocci, 2003, p. 15.

22. A. Berardinelli, art. cit., p. 17 et 27. 
Dans la première moitié des années 1960, l'écrivaine a vécu une crise profonde, à la fois existentielle et littéraire, qu'une partie de la critique a interprétée comme une véritable «césure » dans sa production littéraire ${ }^{23}$. Sans entrer dans le vif de la nature de cette «crise», on s'en tiendra seulement à rappeler que - selon la critique - cette coupure aurait son expression tangible dans une œuvre singulière et totalement différente des précédentes: Le monde sauvé par les gamins. Publié en mai 1968, cet ouvrage marquerait donc le début d'une période qui a été définie - peutêtre trop radicalement - une «deuxième phase » de la poétique d'Elsa Morante, celle qui a vu naître ses deux derniers romans : La Storia (1974) et Aracoeli (1982).

Or, c'est, justement, à ces années de «crise » - que marquent tout particulièrement la passion pour l'œuvre de Simone Weil et la lecture de textes de la pensée orientale - qu'il semble possible de reconduire la lecture de Spinoza aussi, et, surtout celle de l'Éthique. Une analyse de la bibliothèque de l'écrivain semble accréditer cette hypothèse. Il ne s'agit point de la découverte du philosophe hollandais, découverte qui aurait pu se vérifier bien avant, mais de ce qu'on pourrait appeler une intense fréquentation du texte spinozien. En effet, c'est à cette brève première décennie des années Soixante, que remontent les textes de sa bibliothèque «de » et «sur» Spinoza. On y trouve l'édition française des œuvres complètes de Spinoza de la Pléiade ${ }^{24}$; l'Ethica avec le texte latin et la traduction en regard, publié dans les «Classici della Filosofia » de l'éditeur Sansoni en $1963^{25}$; la monographie de Stuart Hampshire, Spinoza (London,

23. C. Garboli, op. cit., p. 20. Sur le plan existentiel, la solitude, après la mort de son jeune ami, le peintre Bill Morrow, le 30 avril 1962 et la rupture définitive avec Sergio Moravia auraient marqué la «crise». Sur le plan thématique, cette période se distinguerait par une prise de conscience de nature idéologique et politique, par la passion pour l'œuvre de Simone Weil et par la lecture de textes de la pensée orientale ; enfin, - sur le plan expressif - par le passage d'un registre essentiellement fantastique à un registre «sapiential » (cf. D'Angeli, op. cit, p. 7). Marco Bardini parle de «fracture» et note, dans les années 1960, la radicalisation chez Elsa Morante, de sa recherche philosophico-existentielle et son rapprochement de la gnose (comprise comme «nihilisme existensialiste ») et du syncrétisme, (Morante Elsa. Italiana, Di professione, poeta, Pisa, Nistri-Lischi, 1999, p. 631, n.18). Une autre partie de la critique a préféré parler d'un itinéraire poétique profondément unitaire en constante évolution: «un itinéraire accompli de façon extraordinairement cohérent, même dans le ton changeant et dans la continuelle évolution de son rapport avec la vie et la page écrite. Un itinéraire qui s'est enrichi de personnage en personnage, de livre en livre » (G. Bettin, Il drago della notte, in Aa.Vv., Per Elsa Morante, cit., p. 220).

24. . Il s'agit du vol. 108 de la Collection NRF, Bibliothèque de la Pléiade, éd. par R. Caillois, M. Francès et R. Misrahi, Paris, 1954, 1962r.

25. Il s'agit de Ethica ordine geometrico demonstrata, edité par G. Gentile (Bari, Laterza,

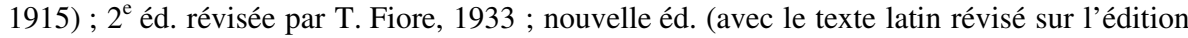
critique Gebhardt) traduite par G. Durante, notes ajoutées et modifiées de G. Radetti, index d'E. Giancotti Boscherini, Firenze, Sansoni, 1963. L'exemplaire d'Elsa Morante est aujourd'hui en possession de Giorgio Agamben, comme il le déclare dans l'ouverture de son court essai : « La festa del tesoro nascosto » [1993], in Id., Categorie italiane. Studi di poetica e di letteratura, Roma-Bari, Laterza, 2010, p. 120-130. 
Penguin Books, 1962); sur la couverture de ce livre, Elsa Morante avait noté : « un excellent essai » et avait ajouté aussi à côté une petite étoile, qui symbolisait, pour elle, l'importance et la prédilection qu'elle accordait à une œuvre; enfin, les Reflexions and maxims de Dagobert D. Runes (New York, Philosophical Library, 1965), dans lequel elle avait souligné avec un feutre rouge certaines voix : Admiration, Animals, Churches, Class hatred, Death, Devil, Immortality, Intellect ${ }^{26}$.

À cette hypothèse chronologique, il serait juste d'associer celle d'une sorte d'immersion d'Elsa Morante dans la lecture de Spinoza, menée avec la même passion dévoratrice et absolue qui lui était propre, dont parlent tous ceux qui l'ont connue. Et il a dû s'agir d'une véritable passion si, dans $L e$ monde sauvé par les gamins, la figure de Spinoza est évoquée de manière hautement emblématique, tandis qu'une forme de « spinozisme » trouve une évidente «profession de foi » dans le roman successif d'Elsa Morante, publié en 1974, La Storia ${ }^{27}$. Notre réflexion se concentrera donc sur ces deux textes.

\section{Le monde sauvé par les gamins : les « Rares Heureux »}

Il est bien difficile de caractériser et de parler du Monde sauvé par les gamins. La forme est celle d'un « recueil de poèmes », dont le protagoniste est un «gamin» polymorphe, nommé il Pazzariello, qui passe à travers une série d'aventures en changeant de corps tout en restant identique dans la substance. En italien Pazzariello, signifie « un peu fou », mais, dans le

\footnotetext{
26. Les Euvres complètes de Spinoza de la Pléiade sont classées (mais sans l'année d'édition) dans le répertoire autographe des livres d'Elsa Morante qu'elle commence probablement dans les années 1950 et qu'elle achève à la fin de 1965. Dans l'exemplaire de la monographie de Stuart Hampshire (qui n'est pas classée dans ce répertoire et qui aujourd'hui se trouve dans le fonds Morante de la Bibliothèque Nationale Centrale à Rome), on peut lire l'observation suivante : «rechercher ce livre : Spinoza's Idea of freedom, Oxford University Press, $1960 »$ et - dans le frontispice (sous le titre) - la note : Samsara = Nirvana . Pour ces renseignements et d'autres sur la biliothèque d' Elsa Morante, voir L. Desideri, I libri di Elsa et le Catalogue des livres déposés auprès du «Fonds Morante » de la Bibliothèque Nationale Centrale à Rome, dans Le stanze di Elsa. Dentro la scrittura di Elsa Morante, a cura di G. Zagra e S. Buttò, Roma, Editore Colombo, 2006, respectivement aux p. 3-9 et 121-148.

27. Lors de la publication de La Storia - bien qu'en employant des tons très différents - Pier Paolo Pasolini («Un'idea troppo fragile nel mare sconfinato della storia », Tempo, XXXVI, $\mathrm{n}^{\circ}$ 30, 19 juill. 1974 et $\mathrm{n}^{\circ}$ 31, 2 août 1974, rééd. in Id., Descrizioni di descrizioni, éd. par C. Chiarcossi, Torino, Einaudi, 1979) et G. Sommavilla (Anarchia e angelologia di Elsa Morante, «Letture», 1974, $\mathrm{n}^{\circ}$ 11, p. 725-738 ; Aracoeli : Elsa Morante, una poetessa tragica del nichilismo, ivi, 1983, p. 33-36 ; Heinrich, Böll ed Elsa Morante : monismo uomo-naturaDio e sue incrinature, en Id., Peripezie dell'epica contemporanea : dialettica e mistero, Milano, Jaca Book, 1983, p. 95-142) ont parlé de «spinozisme». Dans La Storia, Pasolini a vu la présence d'un « syncrétisme » faible qui essaie de fusionner l'Évangile, le spinozisme et l'utopie anarchique, et qui exclut donc toute crédibilité, tandis que Sommavilla a vu dans le «spinozisme » du roman la phase d'une poétique qui se meut vers un nihilisme radical.
} 
dialecte du Sud de l'Italie, il signifie surtout «celui qui joue» et le verbe pazziare signifie «jouer» (le traducteur français a traduit Pazzariello par «Farfada» et pazziare par «faire le fou »). Sous forme de refrain, une phrase revient très souvent que proclame le Pazzariello comme annonce de libération : pure se ci fa tremare / per gli spasimi e la paura / tutto questo, / in sostanza e verità, / non è nient'altro / che un gioco, Même si de tourments et de peur/nous fait trembler / tout ça, / en substance et vérité, / ce n'est rien d'autre qu'un jeu ${ }^{28}$. C'est à cette phrase qu'est confié, dans ce livre, le sens de l'existence, de la «roue de l'être ${ }^{29}$, qui représente hors-temps les mêmes vicissitudes : la naissance, la douleur, la mort. Un sens intensément dramatique, dont toutefois le «gamin», le Pazzariello, parvient à saisir la « légèreté » qui y est cachée.

Elsa Morante, elle-même, définit son livre comme un «manifeste », une «clef magique», un «testament», mais un «roman» aussi et une «autobiographie », qui se comprend «comme l'aventure désespérée d'une conscience qui tend, dans son processus, à s'identifier avec tous les autres vivants de la terre ${ }^{30}$. C'est un texte «inclassable», un pamphlet iconoclaste, burlesque et tragique, où se mêlent le sacré et le profane dans un hymne à la puissance de la Vie ${ }^{31}$, représentée par le Pazzariello dans ses transformations, puissance qui s'oppose au «Pouvoir», qui lui aussi est une entité polymorphe et changeante. Un texte qui «annonce » mai 68 : le refus $\mathrm{du}$ «monde organisé », auquel s'oppose le désir absolu d'une vie sans contrainte, ni ordre établi, ni prison ${ }^{32}$.

Du milieu idéal de ce livre se détache La canzone degli F.P. e degli I.M, titre traduit en français La chanson des $R$. H. et des $N$. M. Le premier sigle indique $i$ Felici Pochi, les Rares Heureux - expression qui rappelle the Happy few de la fin de la Chartreuse de Parme de Stendhal, auteur qu'Elsa Morante aimait beaucoup et dont elle possédait les œuvres en français ${ }^{33}$. Les

28. E. Morante, Le monde sauvé par les gamins, tr. cit., p. 189 (voir aussi p. 195, 267); éd. orig., p. 192 (voir aussi p. 146, 220).

29. P. Dallamano, « Mistico itinerario di Elsa Morante », Paese Sera, 15 juill. 1968, p. 7-8.

20. E. Morante, Le monde sauvé par les gamins, Note introductive, cit., p. 10 (éd. it. cit., p. VI). Les différentes définitions du livre apparaissent en quatrième de couverture.

31. C. Cazalé Bérard, Elsa Morante : poetica e poesia. "Alibi" e "Il mondo salvato dai ragazzini”, in Aa.Vv., Innumerevoli contrasti d'innesti : la poesia del Novecento (e altro), miscellanea in onore di Franco Musarra, Leuven University Press, Leuven, F. Cesati, Firenze, 2007, vol. 1, p. 44 et 47).

32. Le livre a été défini « un acte de subversion anarchique », mais aussi « respect religieux » pour la vie qui est menacée dans son authenticité (C. Bo, «Corriere della sera », cit. dans la Nota introduttiva à Il mondo salvato dai ragazzini, cit., p. VIII), un « joyeux appel aux armes » pour promouvoir une libération intime, "plus radicale et plus subversive », plus profonde que celle qui aurait appartenu au mouvement révolutionnaire, à son tour infecté très vite par la «maladie » du «Pouvoir» (A. Landolfi, «Elsa Morante e l'utopia "impossibile" dei Felici Pochi », Lo Straniero, n 137, 2011, p. 93-94).

33. To the happy fews, c'est la phrase qui achève la Chartreuse de Parme et qui renvoie peut-être à Henry $V$ de Shakespeare : We few, we happy few, we band of brothers (acte IV, scène III) ou aussi à The Vicar Wakefield, de Goldsmith, dont le protagoniste écrit des livres 
Rares Heureux sont les «gamins », c'est-à-dire les «cœurs purs » de l'Évangile, ceux qui ne s'alignent pas sur les autres et qui ne sont ni assimilés ni assimilables, donc exclus et «bannis » de mille façons aussi. Ils représentent le «sel de la terre » de l'Évangile, les vrais « révolutionnaires».

Le second sigle indique gli Infelici Molti, les Nombreux Malheureux. Les Nombreux Malheureux, au contraire, sont ceux qui s'alignent, les adultes, qui vivent à l'abri des prisons aliénantes qu'ils se sont construites à savoir celles du pouvoir sous ses multiples aspects : religieux, militaires, politiques, familiaux. Ils se conforment aux apparences et sont étrangers à toute volonté de compréhension. Ils vivent loin de la vérité et de la beauté, dans la dimension de la mort, ou de la «tristesse ». Apparemment beaux et gagnants, en réalité ils sont tristes et laids, parce que - comme dit la chanson, reprenant ce que Morante avait expliqué dans ses réflexions théoriques - «la REALTA' / è di rado visibile alla gente », la REALITÉ / il est rare que les gens la voient.... ${ }^{34}$.

L'opposition entre les Rares Heureux et les Nombreux Malheureux se décline donc comme une opposition entre la «joie» et la «tristesse », la «réalité » et l' «irréalité » (ou apparence), l'adhésion à la vie et le renoncement à l'authenticité, l'autonomie et l'hétéronomie. Au milieu de toutes ces vicissitudes, on célèbre le sens du divin, l'immanence de Dieu, qui ne peut avoir de visage sans les visages humains qui l'expriment. Le visage de Dieu n'est pas le visage humain, mais il n'existe pas sans le visage humain. Dieu voit seulement à travers les yeux des hommes : "la maison de cet unique / Dieu - dit la chanson - ce sont les vivants, / et si ceux-ci ferment leurs fenêtres, l'habitant de la maison reste aveugle. C'est nous qui devons rouvrir les lumières de nos yeux pour que lui renvoie ${ }^{35}$. Donc, on lira: «Nulle Révélation [...] Nul péché [...] Et nulle grâce spéciale » ${ }^{36}$, voire: nulle religion révélée qui puisse sauver les hommes. Un esprit évangélique radical et laïque - exprimé par une écriture démystifiante, pleine de citations des classiques, reformulées souvent sous forme de parodie nourrit la métaphore du «gamin » comme un état intérieur de lumière, de joie, de vérité, comme condition originelle de l'homme, à laquelle il est difficile, mais il n'est pas impossible de revenir: "Peut-être - lit-on redevenez des petits enfants, enseigne que la dernière / intelligence de la fin / est dans l'identification avec le début ${ }^{37}$.

Mais qui sont les Rares Heureux? Ils sont définis : «des accidents fatals des Mouvements Perpétuels, des graines originelles du Cosmos $»^{38}$,

pour son plaisir et dans l'espoir qu'un jour, les Rares Heureux le lisent (voir le commentaire à la traduction italienne de F. Zanelli Quarantini, Milano, Mondadori, [1980], 2009, p. 534).

34. E. Morante, Le monde sauvé par les gamins, tr. cit., p. 157 ; éd. it. cit., p. 119.

35. Ivi, tr. cit., p. 180 ; éd. it. cit., p. 137-138.

36. $I v i$, tr. cit., p. 50 (éd. it. cit., p. 29).

37. Ivi, tr. cit., p. 180 (éd. it. cit., p. 138).

38. Ivi, tr. cit., p. 158 (éd. it. cit., p. 120). L'«accidentalité » dénote qu'ils n'appartiennent pas nécessairement à une classe ou à une condition sociale, mais qu'ils ont une nature 
c'est-à-dire qu'ils représentent la métaphore de la Vie authentique et du bonheur, dont ils indiquent la rareté et l'accidentalité. Ils sont aussi « indescriptibles », cependant et seulement à titre d'exemple, l'identité de certains d'entre eux est dévoilée. Le poète ambulant de la Chanson des Rares Heureux feuillette une «Encyclopédie universelle» imaginaire et extrait - comme d'une espèce de minuscule Spoon River - quelques épigraphes des Rares Heureux défunts, "de renommée internationale », qui composent une liste provisoire dont la disposition typographique est en forme de croix. Chaque épigraphe indique le nom du défunt, son "mérite », synthétisé en une phrase lapidaire et l'année de sa mort, précédée de son âge. En haut et au centre de la croix, on trouve Spinoza: «BENEDICTUS Spinoza (la fête du trésor caché). Mort banni à l'âge de 45 ans en 1677 ». Parmi les autres personnages qui composent la croix, on trouve les philosophes : Giordano Bruno, Simone Weil, Antonio Gramsci, Platon. En outre, on trouve Jeanne d'Arc, Rimbaud, Mozart, Bellini et Rembrandt.

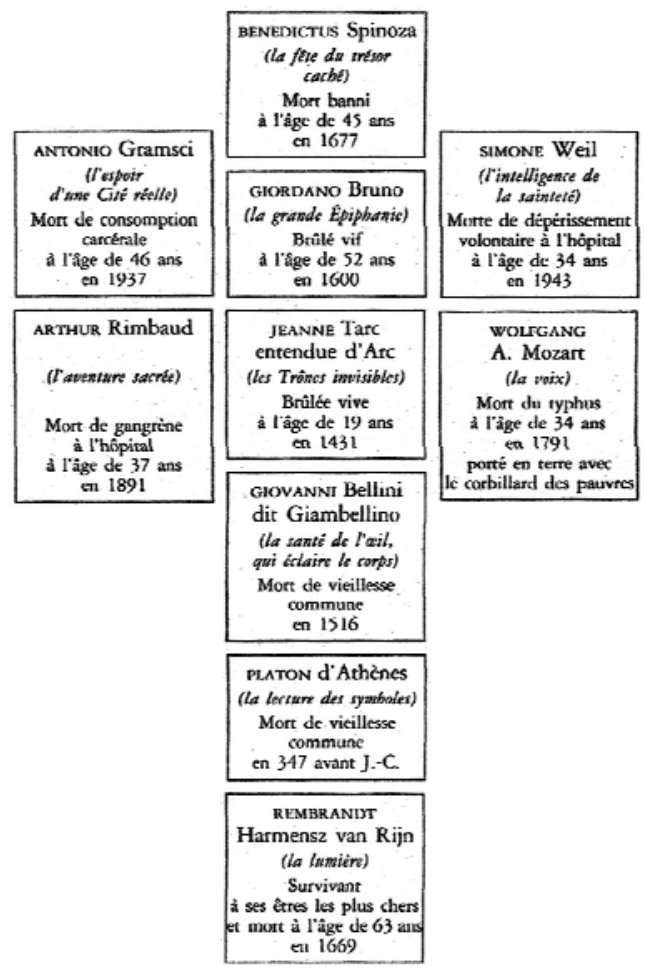

Pourquoi cette disposition en forme de croix et quel sens a la mystérieuse épigraphe qui rappelle Spinoza ? La croix - symbole du salut -

transversale, ils « germent en tout terrain » : le «Bonheur» peut donc appartenir aux «gens cultivés » comme aux « illettrés », le bonheur - dit la chanson - est là « où on n'a pas le vice d'assassiner les prophètes/ni d'exterminer/les poètes » (ivi). 
constitue, ici, une synthèse éloquente de l'idée de « révolution» selon Elsa Morante, dont on a déjà parlé. Il faut noter qu'à l'exception de Jeanne d'Arc - symbole de la puissance visionnaire qu'elle exprimera à travers ses exploits -, tous les autres personnages sont des auteurs d'œuvres d'art, celles que Morante appelait, "chefs-d'œuvre» et qu'elle définissait comme expressions de l'intégralité authentique de l'homme, synthèse de l'éthique et de la beauté. De cette croix salvatrice Elsa Morante « aurait voulu que tout le monde s'en charge ${ }^{39}$.

Les portraits de la croix sont donc des figures de «gamins», de Pazzarielli, et, dans leur ensemble, les mots qui expliquent les personnages de la croix semblent illustrer les possibles déclinaisons d'être des "gamins », à savoir de la capacité de voir, de quelque manière, la « Réalité », qui est «invisible». L' « intelligence » du philosophe, de même que la «voix »du musicien, ou la «lumière » et l'« œil »du peintre, ou, encore, l'utopie éthico-politique, sous-entendent la capacité de percevoir ce qui est caché sous le voile obscur des apparences: c'est-à-dire la «sainteté », le «trésor», les «symboles», l'«aventure sacrée », les «Trônes invisibles » et la «Cité réelle» (cette dernière expliquée, dans la Chanson, en tant que «l'avènement des poésies des fantaisies des sympathies et des authentiques compagnies !!! ») ${ }^{40}$.

Spinoza donc, comme Mozart ou Rimbaud, a été une des «graines originelles du cosmos», une des expressions authentiques de la Vie, de la Beauté, de la véritable Révolution, un « rare heureux», un «gamin », et du gamin il a exprimé, tout particulièrement, une caractéristique : «la fête », à savoir la «joie ».

Son épitaphe dit qu'il est morto bandito (mort banni). Morante semble donc appréhender dans le «bannissement» une marque polyvalente de la biographie de Spinoza. Et en effet Spinoza est d'abord un juif (comme l'était Elsa Morante elle-même par sa mère) et les juifs - les «exclus » par excellence - sont «bannis» de la société civile. Dans La chanson clandestine du Grand Opéra, ils sont définis comme «exclus du compte étant des asociaux barbares inassimilables ${ }^{41}$. Mais Spinoza est aussi le juif «banni » de sa propre communauté, qui s'est constituée en tant que Pouvoir qui opprime et exclut. Enfin, à cause de l'anomalie de sa pensée, Spinoza a été considéré comme «inassimilable » et donc relégué aux marges de toute orthodoxie (religieuse, éthique, politique). Il a attiré sur lui les épithètes de l'exclusion, de la non homologation, de la subversion envers les valeurs constituées, de même que le Pazzariello, lui aussi est «banni», et dont dit la chanson: "C'est un amoral !/ [...] C'est un mécréant / un athée un hérétique un ferment de dissolution $»^{42}$.

39. F. Ramondino, art. cit., p. 55.

40. E. Morante, Le monde sauvé par les gamins, tr. cit., p. 248 (éd. it. cit., p. 197).

41. Ivi, La Chanson clandestine du Grand Opéra, tr. cit., p. 209 (éd. it. cit., p. 162).

42. Ivi, tr. fr. cit., p. 218 (éd. it. cit., p. 170). 
Enfin, sur la croix, Spinoza est commémoré par la phrase «la fête du trésor caché ». Morante le considère donc comme l'emblème d'une intime dimension joyeuse, dont le motif est quelque chose de très précieux, mais de caché, et qui n'est pas visible immédiatement, quelque chose qui fuit au jeu des apparences et de l' «Irréalité ». On peut alors penser que la «fête », synonyme de «joie»- mot-clef de l'éthique spinozienne -, exprime la nature intimement exultante de la connaissance «vraie» que Spinoza a atteinte, celle qui perçoit la "Réalité », c'est-à-dire qui considère chaque chose comme expression particulière de Dieu. Sur son exemplaire de la traduction italienne de l'Éthique, Elsa Morante avait dessiné des symboles sur certaines pages - «des étoiles, des lignes, des points d'interrogation et d'exclamation »- et elle avait réservé une étoile rouge à la sixième définition du Premier Livre - la définition de Dieu -, et au corollaire de la proposition 25 : «Les choses particulières ne sont que des affections des attributs de Dieu, autrement dit des modes, par lesquels les attributs de Dieu, sont exprimés d'une façon définie et déterminée ${ }^{43}$.

Spinoza a donc découvert le trésor de la «connaissance vraie », celle du Deus sive natura, qui est la « Réalité » infinie que l'épaisseur de l'apparence cache. Au prix de l'exclusion, Spinoza a combattu l' «Irréalité », c'est-à-dire l'aliénation de l'intelligence et de l'imagination. Il l'a fait à travers celle que la Chanson indique comme la seule voie possible : un esercizio d'eroica difficoltà, un exercice d'héroïque difficultée ${ }^{44}$. Morante recourt donc à une expression dont il semble presque possible de repérer sinon une paraphrase directe, du moins un écho de la via perardua qui conclut l'Ethique.

\section{La Storia}

Le mot «fête » de l'épitaphe spinozien nous conduit directement au cœur du grand roman successif : La Storia.

C'est l'histoire intensément dramatique d'une toute petite famille romaine qui connaîtra une fin tragique. Ce roman a pour toile de fond la Seconde Guerre mondiale avec son cortège de morts et de ruines, à savoir les événements de l'Histoire, qui sont synthétisés sous forme de brèves chroniques, imprimés en tout petits caractères en marge de chacun des chapitres.

Les chapitres correspondent à chacune des six années de vie du protagoniste, le petit Useppe. Useppe est un petit «bâtard », né du viol d'Ida - une institutrice de trente-six ans déjà mère d'un adolescent de seize

43. La Pléiade, cit., p. 391-392. En ce qui concerne les citations des œuvres de Spinoza, on se réfère toujours à la traduction que Morante possédait. Au sujet des indications concernant les signes présents dans l'exemplaire de la traduction italienne de l'Éthique de Spinoza, cf. G. Agamben, art. cit., p. 131-132.

44. E. Morante, Le monde sauvé par les gamins, La chanson des R.H. et des N.M., tr. cit., p. 159 ; éd. it. cit., p. 121. 
ans - par un jeune soldat allemand. À la fin de l'histoire, déchirante et bouleversante, tous les protagonistes meurent: le Grand Mal, c'est-à-dire l'épilepsie, emportera Useppe, la mère du petit deviendra folle. Toutes les vicissitudes se déroulent dans un contexte populaire, d'extrême pauvreté et de misère.

Et pourtant, bien que le lecteur soit complètement plongé dans le cœur du drame historique et intensément bouleversé par des événements poignants, il garde en lui comme une «saveur» indélébile, qui constitue le message le plus profond du livre : la saveur de la « fête », à savoir la saveur de la Vie, de sa beauté intrinsèque et de son allégresse, que les décombres de l'histoire défigurent d'une façon permanente. De cette allégresse, les deux demi-frères, à savoir les « gamins » Useppe et Nino en sont l'incarnation.

Le nom en entier d'Useppe est Giuseppe Felice Angiolino - cela correspondrait en français à Joseph Heureux (ou Félix) Petit Ange ${ }^{45}$. Son nom exprime sa nature, comme ses yeux «bleus » limpides, qui renvoient à la beauté du ciel, c'est-à-dire à une dimension éternelle, qu'on ne peut réduire aux seules vicissitudes terrestres ${ }^{46}$. Useppe personnifie l'homme originel, pur, sociable de nature, heureux seulement quand il partage ses biens : «Son instinct natif et inextinguible - explique la narratrice - était de partager son plaisir avec autrui $»$. Ses yeux lui montrent un monde totalement merveilleux et féerique, «illuminé de l'intérieur», où aucune forme n'est répugnante ${ }^{48}$ : quand il apprend le mot «étoile», dans son enthousiasme, les « crachats» aussi deviennent des «étoiles » ${ }^{49}$. Il voit les choses au-delà des catégories du bien et du mal ${ }^{50}$ et il se réjouit simplement de la vie telle qu'elle est, et que son regard, «confiant et joyeux », saisit en tant qu' «éternelle et infinie » dans toutes ses expressions particulières : les choses, les animaux et les personnes ${ }^{51}$. Les caractéristiques que Spinoza confie à l' « homme libre », en tant qu'accomplissement d'un parcours de sagesse, appartiennent donc naturellement à Useppe, figure de l'homme que l'Histoire ne réussit pas à défigurer.

Comme Spinoza de la croix du Monde sauvé par les gamins, et comme le Pazzariello, lui aussi est un «banni » : « Ni baptisé, ni circoncis », aucune paroisse ne le revendique ${ }^{52}$; il est «exilé » de l'école à cause de son

45. E. Morante, La Storia, cit., p. 96 ; tr. fr. cit., p. 142.

46. «Son regard, toujours attentif et parlant comme dans un dialogue universel, était, rien qu'à le voir, une vraie joie » et ses yeux « de leurs clins d'œil continuels fêtent sans cesse le monde » (ivi, tr. cit., p. 160 ; éd. it. cit., p. 109).

47. Ivi, tr. cit., p. 761 (éd. it. cit., p. 532).

48. Ivi, tr. cit., p. 179 (éd. it. cit., p. 123).

49. Ivi, tr. cit., p. 176 (éd. it. cit., p. 120).

50. «Sans aucun doute, pour lui il n'existait pas de différences, ni d'âge, ni de beauté et de laideur, ni de sexe, ni sociales » (ivi, tr. cit., p. 265 ; éd. it. cit., p. 185).

51. Useppe «ne voyait pas les choses réduites à leurs aspects usuels, mais comme des images multiples d'autres choses variant à l'infini » (ivi, tr. cit., p. 175 ; éd. it. cit., p. 120).

52. Ivi, tr. cit., p. 174 (éd. it. cit., p. 111). 
comportement; et à la fin de sa très brève vie, il est exclu de la société humaine, parce que les attaques épileptiques le réduisent à «un animal blessé » qui va se cacher : «Et quant aux autres - commente la narratrice quant au reste de la population de la terre - il y avait longtemps qu'il se sentait mis au ban par eux ${ }^{53}$. Et pourtant jusqu'à sa mort, qu'il pressent obscurément, son cœur est capable de se mettre soudain à battre simplement «à cause du plaisir de vivre ${ }^{54}$. Useppe ne sait ni lire ni écrire, mais il est «poète » instinctivement : il compose de courts poèmes, qu'il récite à son chien et qu'il oublie ensuite ${ }^{55}$. C'est donc l'image de l'artiste vrai : libre de tout conditionnement et qui se lie en sympathie avec les choses.

Nino, le frère d'Useppe, est la joie explosive qui excite tout le monde ${ }^{56}$ et qui ne supporte aucun lien, aucune règle. Il se définit lui-même «il re dell'anarchia », le roi de l'anarchie ${ }^{57}$, et crie au monde entier qu'il veut seulement «vivre », «plaire à tout le monde », « dévorer la vie tout entière, et le monde entier, et l'univers entier! Avec ses soleils, ses lunes et ses planètes !!! » ${ }^{58}$. Nino est le « jeune fou » qui cogne instinctivement contre le visage multiforme du Pouvoir - même quand ce dernier revêt les apparences de la Révolution : «EUX - déclare Nino en faisant allusion à ceux qui perpétuent les rapports du Pouvoir et les appliquent à tout - Eux, ils ne le savent pas, [...] comme la vie est belle $! \gg$.

Physiquement aussi Nino reproduit le Pazzariello anarchiste du Monde sauvé par les gamins : les yeux foncés et «les petites boucles emmêlées », selon une iconographie du gamin chère à Elsa Morante, qui semble fonder ensemble la typologie du gamin méridional des quartiers populaires et celle du juif, presque une «fusion » de Masaniello avec Spinoza, tel qu'il nous apparaît dans le portrait présumé du philosophe hollandais ${ }^{60}$.

Tous les deux - Nino et Useppe - sont définis Pazzarielli ${ }^{61}$. Ils incarnent la «fête » de la vie, mais de deux manières différentes: Nino est une

53. Ivi, tr. cit., p. 681, 761 (éd. it. cit., p. 476, 532).

54. Ivi, tr. cit., p. 901 (éd. it. cit., p. 628).

55. Ivi, tr. cit., p. 763 (éd. it. cit., p. 534).

56. «Tout le monde était excité par sa présence joyeuse » (Ivi, tr. cit, p. 509 ; éd. it. cit., p. 354).

57. Ivi, tr. cit., p. 573 (éd. it. cit., p. 403).

58. Ivi, tr. cit., p. 510 (éd. it. cit., p. 354) ; ivi, p. 573 (éd. it. cit., p. 402).

59. Ivi, tr. cit., p. 632 (éd. it. cit., p. 443). Lorsque la narratrice décrira la mort de Nino, elle dira significativement qu'il a été « expulsé de la vie » (ivi, tr. cit., p. 664 ; éd. it. cit., p. 464).

60. Ivi, éd. it. cit., p. 210 (tr. cit., p. 301). Signes caractéristiques du Pazzariello, ce sont les «Cheveux noirs foncés longuets et frisottés, yeux marron et semés d'or »; «par ses boucles de cheveux, par son front, il avait quelque chose de juif... Au moins un quart de sang juif, il devait bien l'avoir » (E. Morante, le monde sauvé par les gamins, éd. cit., p. 166, cfr., p. 184 : tr. fr. cit., p. 213 et 234). Dans La Storia, les juifs du ghetto de Rome sont décrits comme «des petites familles aux cheveux bouclés et aux yeux noirs » (tr. cit., p. 486 ; éd. it. cit., p. 338).

61. E. Morante, La Storia, éd. it. cit., p. 403 (tr. cit., p. 574). L'esprit d'Useppe est défini comme pazzariello (ivi, éd. it. cit., p. 395 ; tr. fr. cit., p. 562 : « esprit follet»). 
« présence joyeuse » instinctive, irrésistible, contagieuse. Useppe est la festa totale del mondo, la fête toute entière du monde, qu'il exprime non point avec des actions et des exploits audacieux, comme son frère, mais avec sa simple existence, avec l'allégresse de tous ses petits membres ${ }^{62}$. Mais c'est l'auteur même qui exprimera en synthèse le sens de la fête des deux personnages à travers les paroles d'un autre personnage-clef du livre : l'ami de Nino durant la Résistance, l'idéologue du livre, le jeune Davide Segré, un juif cultivé, d'origine bourgeoise, au caractère introverti et ombrageux, théoricien de l'anarchie, mais incapable de rester fidèle à son idéal et extrêmement tourmenté. Miné par un trop plein de pensées, et opprimé par le conflit des passions (chez lui - écrit la narratrice - « un loup » et « un faon » cohabitent $^{63}$ ), Davide finira par se donner accidentellement la mort. En s'adressant à Useppe, David lui dit : «Toi et ton frère vous êtes si différents que vous n'avez même pas l'air d'être frères. Mais vous vous ressemblez pour une chose : le bonheur. Ce sont deux bonheurs différents : le sien, c'est le bonheur d'exister. Et le tien, c'est le bonheur... de... de tout. Toi, tu es l'être le plus heureux du monde ; « [...] le seul fait que tu existes - lui confie Davide - me rend heureux $»{ }^{64}$.

Useppe incarne donc le «Bonheur », celui qu'on pourrait définir - en termes spinoziens - le gaudium parfait, la joie qui n'augmente pas. Il représente le regard pur, intact, qui jouit de la simple existence de toutes les choses qui forment le monde, chacune considérée dans sa «perfection», c'est-à dire, sub specie aeternitatis. Nino est, au contraire, la joie explosive, l'irrésistible instinct vital (la cupiditas), c'est le «jeune fou» qui cogne instinctivement contre le visage multiforme du «Pouvoir» (même quand il revêt les apparences de la Révolution) dont il opère le «boycottage systématique $» 65$.

Ce n'est donc pas à travers quelque profession verbale ou doctrinale, mais avec leur être et leur existence mêmes que Nino et surtout Useppe, représentent la puissance et l'exultance de la vie parmi les décombres de l'Histoire, qui est définie, dans le roman : «un mélo grotesque, démentiel, un dépôt d'ordure " ${ }^{66}$. L' "éternelle roue de l'être» du Monde sauvé par les gamins, assume ici les traits angoissants d' " un interminable assassinat », d' « une longue obscénité », réglée sur le "principe immuable», qui veut donc « aux uns le pouvoir et aux autres la servitude ${ }^{67}$. Mais, immanente à ce massacre - qui est raconté dans le roman sans la moindre concession à la consolation - la «Vie» laisse filtrer des traces d'elle-même, des traces de «beauté », que l'auteure réussit à rendre sensible au lecteur à travers les yeux d'Useppe et la faim de vie de Nino.

62. «tripudio [...] di tutte le sue membra » (ivi, éd. it. cit., p. 121 ; tr. cit., p. 176).

63. Ivi, tr. cit., p. 734 et 827 (éd. it. cit., p. 514 et 578).

64. Ivi, tr. cit., p. 743, 857 (éd. it. cit., p. 520, 599).

65. E. Morante, Le monde sauvé par les gamins, tr. cit., p. 235 (éd. it. cit., p. 170).

66. E. Morante, La Storia, tr. cit., p. 848 (éd. it. cit., p. 592).

67. Ivi, tr. cit., p. 929, 836, 13 (éd. it. cit., p. 647, 584, 7). 
La «Vie» naît et meurt sans cesse dans l'« histoire», dans l'« éternelle roue de l'être », mais elle est infiniment plus riche que l'Histoire. C'est pour ça qu'elle ne peut être "racontée » à travers un réalisme qui s'appuie sur la « photo » et sur le «document». Le recours stylistique, qui consiste à avoir fait précéder la «chronique » de l'Histoire en marge de chaque chapitre de l'histoire objet du roman, exprime le refus de faire coïncider la vie avec la phénoménologie historique, et la «Réalité » avec la dimension du temps.

L'attitude «anti-empiriste » et « antipositiviste» d'Elsa Morante, qui refuse d'assimiler la «Réalité » à la tuerie de l'histoire, trouve son point de force dans le langage, auquel elle attribue un rôle fondamental : la restitution de la vision de la «Réalité » au-delà des apparences est alors confiée au choix minutieux des mots. Si le «réalisme » est - selon Elsa Morante - la capacité de l'artiste d'exprimer l'inépuisable, au-delà du fini, de dévoiler le sens caché des apparences et de l'universaliser à travers des symboles, l'écrivain pourra réaliser cet artifice seulement en s'attachant à soigner extrêmement et minutieusement ses mots. Elle écrit :

Avec le sentiment aventureux et presque héroïque de celui qui cherche un trésor souterrain, il devra alors chercher cette unique parole, et aucune autre, qui représente l'objet précis de sa perception, dans sa réalité. Justement cette parole est la vérité, voulue par le romancier [...]. C'est l'exercice de la vérité qui conduit à l'invention du langage, et non pas le contraire [...]. Et plus le romancier sera proche de sa maturité parfaite, plus son langage se fera simple et limpide dans ses couleurs. Et l'art le plus difficile, pour le romancier, c'est de refléter dans son propre langage la limpidité de la vérité ${ }^{68}$.

C'est dans le plein de cette maturité qu'Elsa Morante construit très clairement et efficacement dans La Storia le lexique de la «fête », grâce auquel elle raconte le drame de l'Histoire sans jamais céder au fabuleux, tout en réussissant à garder merveilleusement le registre «réaliste». La construction savante de ce lexique se réalise à travers deux modalités fondamentales : 1) la fréquence des lemmes qui expriment la joie et la lumière ; 2) le recours spécifique à l' «altération » des noms, afin d'exprimer l'affection, la petitesse, la grâce, la tendresse et la sympathie. Cette dernière caractéristique, spécifique de l'italien - qui permet d' «altérer » n'importe quel nom de plusieurs façons, en créant des suggestions affectives particulières, mais aussi des effets musicaux sur le plan étroitement linguistiques - contribue à provoquer un sentiment de contraste strident entre, d'un côté, la tragicité presque insupportable des événements historiques et l'extrême misère des lieux dans lesquels ils se déroulent, et - de l'autre - la «Vie» qui se défigure de plus en plus tout en léguant, comme trace impérissable, un sentiment profond de tendresse ${ }^{69}$.

68. E. Morante, Sur le roman, tr. cit., p. 57-58 (éd. it. cit., p. 56).

69. Une page extrêmement significative de ce que je viens de dire est, dans La Storia, celle qui parle de la visite d'Ida, la mère d'Useppe, au ghetto juif, après la déportation en masse des habitants : dans le vide total des lieux, les objets (portes, échelles, langes, etc.) sont décrits à 
Mais c'est surtout l'emploi - abondant, sans toutefois ne jamais être ni affecté ni déplacé - des mots qui expriment la fête, c'est le recours à la construction systématique d'un lexique de la joie ${ }^{70}$, qui permet à l'écrivain de garder intacte chez le lecteur, la « saveur » de la Vie, «le trésor caché », la « réalité » infinie mais invisible, au fur et à mesure que se déroulent ces tragiques vicissitudes.

Dans le lexique de l'allégresse le lemme le plus fréquent est festa (fête) avec tous ses dérivés festante, festoso, festivo, même festantissimo (qui, sont traduits en grande partie, par les mots joie, joyeux, ou par des périphrases comme : en grande liesse). Au mot festa on peut ajouter d'autres substantifs comme gloria, allegria, entusiasmo, meraviglia, fervore, fiducia, bellezza, riso, (gloire, allégresse, allégresse particulière, merveilleuse allégresse, gaieté, enthousiasme merveille, ferveur, confiance, beauté, beauté suprême, rire, etc.). On peut ajouter aussi des termes qui renvoient à la lumière et à la luminosité, comme sfolgorio (éclat, étincellement), les verbes illuminarsi (s'illuminer), risplendere (resplendir); les adjectifs: radioso (radieux), lucente (resplendissant), colorat (coloré) etc. Encore, une vaste gamme d'ajectifs : gioioso, giulivo, gaudioso, esilarato (joyeux), allegro (allègre), beato (béat), gaio (gai), trionfante (triomphant), esultante (exultant) fiducioso (plein de confiance), favoloso, sognante (rêveur), incantat (ravi), divino (divin), fantastico (fantastique), strepitoso (sensationnel), melodioso (mélodieux), palpitante (palpitant), etc. Enfin on peut ajouter des expressions redondantes, comme risplendere di fervore (resplendir de ferveur), multipla fantasmagoria (multiple fantasmagorie), tripudio supremo (au comble de l'allégresse), festa totale (la fête toute entière du monde), allegrezza meravigliosa (merveilleuse allégresse) ${ }^{71}$, etc.

Le lexique de la fête (et l'emploi intensif des diminutifs et des mots altérés au sens affectif) s'accumule principalement tout autour de la figure d'Useppe, dont les risatine fresche e irrequiete (les petits rires frais et

travers les yeux d'Ida par des noms « altérés », pour exprimer le sens de la vie effacée, violée, mais encore présente : calzoncini (pantalon court d'enfant) fasciole (langettes), portoncino, uscioli (petites portes d'entrée), cordicelle (cordelette), scaluccia (petit escalier), manina (menotte), targhetta (plaquette), bestiola (bestiole) (E. Morante, La Storia, éd. cit., p. 340343, passim ; tr. fr. cit., p. 487-489, passim).

70. G. Sommavilla («Anarchia e angelologia di Elsa Morante», in Letture, cit., p. 726) met clairement en évidence la fonction du lexique dans La Storia et souligne, en particulier, la présence d'adjectifs employés «de façon absolue » qui ont la fonction d'exalter certaines réalités extérieures et les états d'âme correspondants tout en trahissant le sentiment fondamental du livre, voire celui de «fête absolue». L'ontologie qui correspond à un tel sentiment est - selon Sommavilla - un « ontologisme passionné et glorifiant », un « monisme ontologiste », qui cohabite avec une vague forme de «transcendance». Toutefois une telle sorte d'ontologie implique un nihilisme, qui deviendra de plus en plus tragique (cfr. G. Sommavilla, Elsa Morante, una poetessa tragica del nichilismo, cit. Heinrich, Böll ed Elsa Morante, cit.).

71. E. Morante, La Storia, éd. cit., p. 88, 122, 443, 121, 537 (tr. cit., p. 270, 178, 632, 176, 768). 
inquiets), comme cascatelle (de petites cascades) imprègnent tout le roman, et en constituent l'arrière-fond ${ }^{72}$.

Dans une perspective narrative de documentation ou «néo-réaliste ${ }^{73}$, c'est-à-dire au niveau de ce que Morante appelle les « apparences », Useppe est seulement un petit analphabète, qui a des problèmes d'apprentissage et de langage, un enfant chétif, exclu par les gens dits «normaux ». Mais, dans la «Réalité », chez lui on célèbre la «fête » de la «Vie », c'est-à-dire un Amor Dei spontané, que la tristesse effleure à peine, sous forme d'une ombre qui se glisse dans le bleu de ses yeux. C'est donc exclusivement grâce à l'usage savant et méthodique du lexique de la joie et - sans faire recours au soutien d'histoires réconfortantes - que l'écrivaine crée le sentiment de la fête. À travers l'emploi contrôlé et concerté des mots de la fête, elle confie au petit Useppe la capacité de saisir spontanément toutes les choses sous un même statut ontologique (c'est-à-dire en termes spinoziens, en tant que « modes » de la nature infinie) et donc d'être toujours joyeux.

Useppe, symbole d'une nature humaine que l'Histoire défigure, est le déterreur du "trésor caché », donc l'ontologue inconscient du roman. Dans les dernières pages du livre, ce sera Davide, l'intellectuel, qui fera une traduction en termes conceptuels des poèmes de Useppe et exprimera verbalement l'ontologie qui est impliquée dans son allégresse, professant explicitement et ouvertement la philosophie de Spinoza. Un jour, après avoir écouté les poèmes du petit garçon, Davide lui explique qu'ils «parlent tous de Dieu », parce qu'ils mettent en liaison toutes les choses et que «Le seul Dieu réel se reconnaît à travers les ressemblances de toutes les choses. Où que l'on regarde, on découvre une unique empreinte commune [...] Dieu, c'est-à-dire la nature... » (il faut noter que l'expression Dio, ossia la natura..., est écrite en italique, comme pour indiquer qu'il s'agit d'une citation $)^{74}$. «Pour un esprit religieux [...] - poursuit Davide - il n'y a pas d'objet, fût-ce même un ver ou un fétu de paille, qui ne rende semblablement témoignage de DIEU ! ${ }^{75}$.

Cette traduction en termes conceptuels des poèmes d'Useppe est confiée à Davide, parce que, comme Useppe, lui aussi, avant de basculer dans le «trop plein des pensées »- était un «poète ». En effet, il se souvient d'un de ses poèmes d'adolescent qui célébrait l'identité de la «poésie» et de la « vraie révolution » : «La grande Révolution est enseignée par l'air / qui se donne à toutes les respirations et les reçoit toutes. / Elle est chantée par la mer, notre sang infini, / qui dans chacune de ses gouttes réfléchit le soleil tout entier! De même toute pupille humaine réfléchit la lumière tout

72. Ivi, éd. cit., p. 597, p. 599 (tr. cit., p. 856, 858).

73. «Il me semble, à moi - écrivait Elsa Morante - , que certains produits du [...] néoréalisme contemporain, sont de suprêmes exemples de non-réalisme, de non-engagement, et d'évasion » (Sur le roman, cit., p. 51).

74. E. Morante, La Storia, tr. cit., p. 748-749 (éd. it. cit., p. 523-34).

75. Ivi, tr. cit., p. 748-749 (éd. it. cit., p. 525). 
entière ! $»^{76}$. À travers Davide, on sait donc que l'ontologie d'Useppe n'est pas seulement un vague sentiment romantique de la nature, mais elle est intrinsèquement « révolutionnaire ».

Davide poursuivra et articulera sa profession de panthéisme révolutionnaire en un long monologue (qui emboîte le pas à Dostoïevski) qu'il proclamera dans une petite taverne à des auditeurs dérisoires et distraits. Il parlera alors de coscienza, conscience humaine, - terme qu'on pourrait faire correspondre à l'intelligere de Spinoza - et il l'expliquera comme «fête de Dieu »: «La créature humaine - révèle Davide - signifie : la conscience. C'est la Genèse. La conscience est le miracle de Dieu. Elle est Dieu! Ce jour-là Dieu dit: Voici l'homme! Et puis il dit: Je suis le fils de l'homme! Et ainsi finalement il se repose et se réjouit. Mais la conscience, dans sa propre fête, est une et totale: dans la conscience, il n'existe pas d'individus séparés. Et dans la réalité, il n'existe aucune différence entre une créature humaine et l'autre ${ }^{77}$.

Davide ne se limite donc pas à affirmer que la conscience humaine, l'intelligence de la réalité, est la plus haute manifestation de Dieu, mais il ajoute qu'elle est Dieu-même qui fa festa (se réjouit), parce que dans la conscience «dans sa propre fête », à savoir dans la «Réalité», il n'y a pas de séparation entre les individualités.

$\mathrm{Si}$ on en reste prudemment sur le plan des simples assonances - sans aucune prétention de parler de sources - dans les mots de Davide, il est possible de distinguer un rappel aux dernières Propositions de l'Éthique de Spinoza, et, surtout, à la démonstration de la proposition 35, où Spinoza parle de Dieu qui gaudet - qui jouit, de la propre perfection infinie -, après avoir précisé, que l' «intellect» et la «joie » de Dieu appartiennent non pas à sa natura naturans, à sa nature infinie, mais à sa natura naturata, à savoir aux intellects des hommes (modes de Dieu) quand ils se constituent comme unité éternelle et intemporelle : l'intellectus o idea Dei.

Le Dieu de Davide/Morante «s'est fait chair», à savoir il «vient au monde », où il «fait fête» (ce sont toutes les expressions que Davide emploie comme équivalentes) dans la «conscience » de l'homme, où toutes les choses ont la même dignité. Son panthéisme donc ne s'épuise pas dans la perception de l'unité intime de la nature, mais, comme celui de Spinoza, il comporte la fraternité humaine aussi et le partage commun. La «conscience » donc est intrinsèquement révolutionnaire : elle ne doit pas « choisir» la révolution, elle-même l'est, tout naturellement, elle renverse les rapports de pouvoir en générant la spontanéité du partage et en dissipant l'aliénation de l'imagination.

La conscience est «immortalité » aussi, à savoir la conscience de l'appartenance éternelle de toute chose à une même racine vitale. Davide déclare en effet : «De fait, on dit : dieu est immortel, précisément parce que

76. Ivi, tr. cit., p. 751 (éd. it. cit., p. 525).

77. Ivi, tr. cit., p. 815 (éd. it. cit., p. 569). 
l'existence est une, la même, chez toutes les créatures vivantes. Et le jour où la conscience sait cela, que reste-t-il alors à la mort? Pour ce tout qui est un seul la mort n'est rien : est-ce que la lumière pâtit si toi ou moi fermons les paupières? ! ${ }^{78}$. Sur le plan de l'éternel, dans la conscience du «tousun », la mort donc n'existe pas. Mais sur le plan du temps, de l'histoire, la mort affiche, sans pitié, la victoire. Aucune fin heureuse, aucun «Monde Nouveau », comme croit au contraire fermement le vieux tavernier Remo, qui croit à la «vérité scientifique » de la doctrine communiste ${ }^{79}$. C'est la négation de tout ratio qui tisse les fils de l'histoire : tous les personnages du roman qui essaient de donner un sens à l'Histoire à travers une profession de foi ou une idéologie, sont vaincus. Chaque philosophie de l'Histoire, chaque perspective téléologique (aussi bien laïque que religieuse) se montre impuissante. Et pourtant la «réalité » de la «Fête» ne cesse ; au contraire elle semble toucher son sommet expressif exactement dans les dernières pages, celles qui racontent la mort d'Useppe et, avec elle, celle de tout espoir dans les «Trônes invisibles », à savoir la possibilité de réalisation d'un projet éthico-politique qui ne soit pas infecté par les mécanismes du pouvoir.

Comme Nino, Useppe aussi meurt encore «gamin». Quand il commence à avoir une perception confuse du «mal»- de la mort de son frère Nino (qu'on lui a cachée), de la «maladie » psychologique et morale de Davide et, surtout, de sa maladie à lui -, c'est alors que naît en lui la grande question, qu'il pose à sa mère, dans son langage enfantin : « $A^{\prime}$ ' mà... pecché ?», «M'man... pourquoi ? ${ }^{80}$. Sa mère ne sait pas quoi lui répondre, elle-même détruite par la douleur, mais c'est la «nature » qui le fait, c'est-àdire son indomptable joyeuse perception de la vie, ou - pourrait-on dire c'est Dieu même qui le fait, qui s'exprime dans sa conscience absolument «pure », intacte.

La réponse lui est révélée dans un lieu «enchanté», une cabane que les arbres forment et qu'il a découverte le long du fleuve dans ses derniers mois de vie. C'est son «Paradis» (et ce sont des vers de la Divina Commedia de Dante que Davide récite qui confirment indirectement qu'il s'agit du Paradis ${ }^{81}$ ). Un jour, dans son «Paradis », Useppe - qui connaît le langage des animaux - entend les petits oiseaux qui chantent une chansonnette : $\grave{E}$ uno scherzo / è tutto uno scherzo. Dans la traduction française, on peut lire : C'est un jeu / un jeu / rien qu'un jeu ${ }^{82}$, à savoir, la reprise textuelle du refrain de consolation $d u$ Monde sauvé par les gamins : «tout ça, / en substance

78. Ivi, tr. cit., p. 817 (éd. it. cit., p. 571).

79. Ivi, tr. fr. cit., p. 691 (éd. it. cit., p. 483).

80. Ivi, tr. fr. cit., p. 714 (éd. it. cit., p. 550).

81. Davide cite les vers du Paradis de Dante: «...E vidi lume in forma di riveralfluvido di fulgore intra due riveldipinte di mirabil primavera. Di tal fiumana uscian faville vive!» (ivi, éd. it. cit., p. 527 ; tr. cit., p. 754: «...Et je vis une lumière en forme de rivière,/éblouissante entre deux rives/que colorait un merveilleux printemps./De ce fleuve sortaient des étincelles vives »). L'hortus d'Useppe est une île heureuse comme celle de Procida de L'isola di Arturo, et - comme cette dernière -, c'est une dimension de l'âme.

82. E. Morante, La Storia, éd. it. cit., p. 509 (tr. cit., p. 728). 
et vérité, / ce n'est rien d'autre qu'un jeu ». Il est question bien sûr d'une nouvelle version du refrain, mais en italien le terme gioco (jeu) a été remplacé par scherzo, mot qui contient une nuance moins joyeuse et teintée d'une ironie un peu amère. Une nuance significative de la parabole de la narration d'Elsa Morante vers un « réalisme » aux tons progressivement plus sombres ${ }^{83}$.

On a interprété la chanson qu'Useppe entend comme la négation de la consistance ontologique $\mathrm{du} \mathrm{mal}^{84}$, mais je pencherais davantage vers la vision du reflet d'un état de l'âme. La cabane d'arbres, le Paradis, représente un hortus conclusus symbolique, un état de l'âme d'Useppe, chez qui la douleur - même dans son énigmaticité insupportable - ne détruit pas sa joie d'être au monde, la «Fête» de la «Vie». Dans le langage qui est propre au «gamin», comme dans Le monde sauvé par les gamins, le refrain exprime l'invincible perception de la «fête » à l'intérieur du mal de l'Histoire. En termes spinoziens, on parlerait de meditatio vitae. Morante semble ainsi livrer au poète-enfant Useppe encore une des caractéristiques de l' «homme libre » de Spinoza : le regard tendu vers l'être, vers la vie. "L'homme libre - affirme la proposition 67 de Ethica, IV - ne pense à rien moins qu'à la mort, et sa sagesse est une méditation non de la mort, mais de la vie $»^{85}$.

Cette attitude naturelle d'Useppe trouve une formulation poétique émouvante dans une des plus belles pages du livre, celle où - toujours dans le cadre de leur petit Paradis - la chienne Bella berce le petit Useppe à bout de forces, lui rappelant la «beauté infinie » de toutes les créatures vivantes, prises dans leur singularité, évoquées chacune par leur nom: les chiots qu'elle a perdus, son ancien maître qui a fini en prison, Nino, le frère qu'Useppe a tant aimé, Useppe même, Ida, sa mère, Scimò, l'ami de ses derniers jours, Davide, etc. ${ }^{86}$ Le traducteur a traduit en français l'adjectif infinito par "suprême », terme très efficace sur le plan linguistique, mais je pense qu'Elsa Morante tenait tout particulièrement au mot «infini », et l'employait selon le sens que lui donne Spinoza, comme capacité qu'a chaque chose d'être l'expression d'une réalité infinie et donc d'une richesse inépuisable. On peut noter aussi que l'adjectif «infini» est souvent utilisé dans le roman pour qualifier les sentiments joyeux d'Useppe: exultance infinie, joie infinie, fête infinie, etc.

À la fin de la «berceuse » de Bella, on peut lire : «Useppe eut un rire satisfait, car, à la vérité, sur ce sujet des beautés l'accord entre la chienne et lui était total. Pour lui, géants ou nains, gueux ou dandys, décrépitude ou

83. Un « réalisme » qui prendra forme dans le dernier roman, Aracoeli, dans lequel le lexique de la fête cède le pas à celui de la «tristesse », mais pas du désespoir. Lexique auquel Elsa Morante apportera le même soin et la même recherche minutieuse. Elle écrira sur la première page du manuscrit du livre : « una favola d'amore » (voir les images des manuscrits qui se trouvent dans le volume Le stanze di Elsa, cit.).

84. G. Sommavilla, Anarchia e angelologia di Elsa Morante, cit., p. 733-735.

85. Trad. cit., p. 603.

86. E. Morante, La Storia, tr. cit., p. 796-798) (éd. it. cit., p. 556-557). 
jeunesse, cela ne faisait aucune différence ${ }^{87}$. Si «en apparence »- ou «dans l'irréalité » - Bella est seulement un gros chien berger, au poil sale (les enfants l'appellent Pelozozzo/Poil cracra), qui accompagne un petit enfant malade et seul dans ses vagabondages le long du fleuve, la caméra idéale du poète, capable de «zoomer» et pénétrer le secret des choses, dévoile «son âme propre et très pure ${ }^{88}$, qui se syntonise avec celle d'Useppe sur la longueur d'onde d'une scientia intuitiva spontanée, qui leur montre les choses sub specie aeternitatis et les fait jouir.

Si ce sont seulement les «gamins », i Pazzarielli, les «petits enfants » de l'Evangile cité en épigraphe au roman (Luc.10,21), qui voient la «Réalité », la vraie implication dramatique du roman semble résider à circonscrire cette vision et la «fête » qui l'accompagne à une enfance réelle et non point seulement métaphorique, comme elle semblait l'être dans $L e$ monde sauvé par les gamins. Nino et Useppe ont déterré, chacun à leur façon, le sens festante, joyeux, de la vie, l'éternel : Nino par sa faim de vie, Useppe par sa «contemplation» de l'éternel. Et tous les deux sont arrachés à la vie encore «gamins », avant que l'histoire n'ait pu défigurer la nature : leur mort semble donc être nécessaire afin que leur « fête » soit intacte.

La dissolution de la métaphore du «gamin » devient manifeste chez Davide - le troisième "gamin » protagoniste du roman. Chez lui le sens de la «fête » qu'il nourrissait depuis son enfance, quand il pensait au bonheur comme « destin des hommes », se dénature, à partir du moment où il devient une « doctrine », une « idée », qui le fait basculer dans un conflit affectif, et jette sa puissance vitale dans le chaos, en le conduisant à l'autodestruction ${ }^{89}$. La mort de Davide est très différente de celle de Nino et d'Useppe : c'est une libération du conflit qui a déjà tué Davide, le «gamin», à savoir celui qui «sentait toutes les cellules de son propre corps tendre [tendere] vers le bonheur ${ }^{90}$. C'est donc avec une nuance d'amère désillusion que la

87. Ivi, tr. cit., p. 797 (éd. cit., p. 557).

88. Ivi, tr. cit., p. 678 (éd. it. cit., p. 474). L'âme de Bella est totalement semblable à celle d'Useppe, au-delà des différences d'espèce, que Morante ne reconnaissait pas. C'est justement sur la conception de l'animal que l'écrivain exprimait ouvertement son désaccord avec Spinoza. Dans son exemplaire de l'Éthique, en marge de la scolie I de la prop. 37 de la Quatrième partie de l'Éthique - c'est-à-dire là où Spinoza affirme la diversité entre la nature humaine et la nature animale, et la supériorité du droit humain sur le droit des animaux - elle note : «Oh Baruch! Je regrette pour toi, mais dans ce cas, tu n'as pas COMPRIS » (G. Agamben, art. cit., p. 132).

89. «Personnellement - commente la narratrice - je pense que, de nature, David Segré aimait trop la vie pour s'en défaire consciemment d'un jour à l'autre » (La Storia, tr. cit., p. 891 ; éd. it. cit., p. 621). Il s'agit de mots qui suggèrent l'idée de l'extranéité du suicide au conatus vivendi, en accord avec Spinoza qui, dans la Quatrième partie de l'Éthique, écrit : «Personne, dis-je, d'après la nécessité de sa nature, mais toujours contraint par des causes extérieures [...] ne se donne la mort », (pr. 20, sch. ; tr. cit., p. 563). Davide ne veut pas se tuer, mais sa mort accidentelle, bien différente de celle de Nino et d'Useppe, est une libération du conflit qui a déjà tué le «gamin » Davide.

90. E. Morante, La Storia, tr. cit., p. 586 (éd. it. cit., p. 411-412). Il faudrait souligner la présence significative du verbe tendre (tendere), référé à l'être dans son entier, comme pour exprimer le conatus à la vie qui s'inscrit dans chaque individu. Il est toutefois impossible de 
narratrice commente : «À la vérité [...] son bonheur aurait pu se chanter en ces quelques mots : il avait dix-huit ans ${ }^{91}$. Si donc, adolescent, Davide pensait que le bonheur était connaturel au destin humain, ensuite, «vaincu par des causes externes »- dirait-on avec Spinoza ${ }^{92}$ - il a fini par changer d'idée, par assigner le bonheur à un état «angélique», qui n'a rien à voir avec la vie sur la terre. Avant de mourir, il dira à Useppe-Angiolino : «Toi, tu es trop gentil pour ce monde, tu n'es pas d'ici. Comme on dit : le bonheur n'est pas de ce monde ${ }^{93}$.

L'aspect menaçant des spirales métamorphiques et omnivores du Pouvoir qui sont décrites dans le roman précédent, semblent marquer ici une victoire absolue avec la mort prématurée d'Useppe, mais de Nino aussi et de $f e u$ le «gamin» Davide. Le Pouvoir a réalisé celle que Davide, avait prophétisée et définie dans son monologue, «la pire violence contre l'homme », c'est-à-dire «la dégradation de l'intellect » ${ }^{94}$, faisant reculer l'humanité - tachée d'effarantes horreurs - au-delà du degré animal ${ }^{95}$. Dans les dernières lignes de la «chronique » du $\mathrm{XX}^{\mathrm{e}}$ siècle qui met fin au livre on lit que cette dégradation continue aujourd'hui aussi, sous forme - je cite - $d$ ' "une "culture" inférieure, servile et dégradante, qui corrompt le jugement et la créativité humaine, interrompt toute réelle motivation de l'existence ${ }^{96}$.

L' «exercice d'héroïque difficulté », la via perardua des Rares Heureux, la recherche du vrai bonheur semble donc s'annoncer dans le roman comme de plus en plus rare. Et pourtant pas impossible. La «chronique» du $\mathrm{XX}^{\mathrm{e}}$ siècle finit par une expression entre guillemets: « ...et l'Histoire continue... », qui indique l'éternelle répétition de la tuerie, des mécanismes du Pouvoir. Mais ce ne sont pas les derniers mots du livre, qui - au contraire - finit par une phrase de Gramsci : «Toutes les graines n'ont rien donné sauf une: je ne sais pas ce qu'elle peut être, mais c'est probablement une fleur et non une mauvaise herbe ${ }^{97}$. " Graines originelles du Cosmos » avaient été définis les Rares Heureux du Monde sauvé par les gamins. La « Réalité »-dans le sens qu'Elsa Morante a donné à ce mot - ne meurt pas sous les décombres de l'histoire, mais elle reste, enfouie sous ces derniers, comme un «trésor caché », que le romancier est capable de communiquer au lecteur.

savoir si on se trouve en présence d'un écho spinozien, indirect aussi. On se limite, encore une fois, à signaler une assonance.

91. E. Morante, La Storia, tr. cit., p. 597 (éd. it. cit., p. 419).

92. Spinoza, Ethica, IV, pr. 20, sch., tr. cit., p. 563.

93. E. Morante, La Storia, tr. cit., p. 744 (éd. cit., p. 520).

94. Ivi, tr. cit., p. 875 (éd. it. cit., p. 611). « La connaissance est l'honneur de l'homme », dit Davide (ivi, tr. fr., p. 873 ; éd. it. cit., p. 609).

95. Ivi, tr. cit., p. 821 (éd. it. cit., p. 574).

96. Ivi, tr. cit., p. 939 (éd. it. cit., p. 655).

97. Ivi, tr. cit., p. 941 (éd. it. cit., p. 657). 
On a dit au début que La Storia laisse au lecteur une saveur de «fête », un sentiment vital. Par ce terme, je n'entends pas une émotion superficielle et passagère, mais une forme de sagesse, de sapientia, mot qui, comme on le sait, a la racine de sapere, avoir la saveur. C'est un sentiment aussi où se fondent la beauté et l'éthique, c'est-à-dire la caractéristique qu'Elsa Morante attribuait aux «chefs d'œuvre »; un sentiment capable de provoquer « une augmentation de vitalité », ce que - selon elle - seules les véritables œuvres d'art sont capables de faire ${ }^{98}$. Le jugement qui a été rédigé le lendemain de la publication du roman, par une illustre lectrice, Natalia Ginzburg, l'exprime parfaitement : « [...] tout ce que j'avais connu et aimé dans ma vie pénétrait dans une nouvelle dimension plus élevée. Parce que la grandeur nous fait devenir plus intelligents, meilleurs et plus justes, et nous donne une idée juste et généreuse de la réalité dans laquelle nous vivons ${ }^{99}$.

Pour conclure ces réflexions avec une autre suggestion spinozienne, on pourrait ajouter qu'il ne serait trop audacieux de voir dans La Storia un parcours de sagesse, intime, profond, semblable à celui que nous offre l'Éthique de Spinoza. Un parcours à la recherche du bonheur. Et, comme Spinoza, pour construire ce parcours, Elsa Morante a eu grand soin de travailler méthodiquement sur la précision des mots et le monde des affects, comme ses cahiers de notes et les manuscrits le démontrent ${ }^{100}$. Comme romancier, ou plutôt «poète», elle s'est efforcée de discipliner son imagination au service de l'intelligence de la réalité, c'est-à-dire au service d'une compréhension des choses qui s'ouvre toujours sur l'infini et donc qui ne se conclut jamais ${ }^{101}$.

98. E. Morante, Sur le roman, tr. cit., p. 74 (éd. it. cit., p. 72).

99. N. Ginzburg, Elzeviri, « Corriere della sera », 30 juin 1974, p. 3.

100. «Elsa mettait une très grande attention à choisir les mots. Elle considérait tous les mots impropres comme une trahison du réel et de la mission du poète. [...] Une nuance imperceptible dans le choix d'un mot et dans son association à d'autres peut renverser et fausser les sentiments, les pensées, les émotions »(F. Ramondino, art. cit., p. 52). Dans les cahiers où elle préparait ses romans, Elsa Morante dressait des listes de mots et traçait les profils psychologiques de chaque personnage (voir, à ce sujet, la documentation dans le volume Le stanze di Elsa, cit.)

101. Dans ses réflexions sur le roman, Elsa Morante avait écrit : «Le romancier, à l'égal d'un philosophe-psychologue, présente dans son œuvre un système du monde et des relations humaines complet, et qui lui est propre. Sauf que, au lieu d'exposer son système en termes de raisonnement, il est entraîné par sa nature à le représenter dans une fiction poétique au moyen de symboles narratifs. Tout roman, donc, pourrait, chez un lecteur attentif et intelligent [...] être traduit en termes d'essai, et d'"œuvre de la pensée" » (Sur le roman, tr. cit., p. 47 ; éd. it. cit., p. 46-47). 